\title{
Sudden Flux Change Studies in High Field Superconducting Accelerator Magnets
}

\author{
S. Feher, B. Bordini, R.Carcagno, A. Makulski, D.F. Orris, Y.M. Pischalnikov, C. Sylvester, \\ M. Tartaglia, J.C. Tompkins, A.V. Zlobin
}

\begin{abstract}
As part of the High Field Magnet Program at Fermilab many magnets have been tested which utilize multi strand Rutherford type cable made of state-of-the art $\mathrm{Nb}_{3} \mathrm{Sn}$ strands. During these magnet tests we observed sudden flux changes by monitoring coil voltages and the magnetic field close to the magnets. These flux changes might be linked to magnet instabilities. The voltage spike signals were correlated with quench antenna signals, a strong indication that these are magnet phenomena. With a new high resolution voltage spike detection system, we were able to observe the detailed structure of the spikes. Two fundamentally different signal shapes were distinguished, most likely generated by different mechanisms.
\end{abstract}

Index Terms-Accelerator, Magnet, High Field Dipole, $\mathrm{Nb}_{3} \mathrm{Sn}$

\section{INTRODUCTION}

$\mathrm{I}_{\mathrm{p}}^{\mathrm{N}}$ NSTABILIES in superconducting magnets can result in premature quenching. Many of the Fermilab built Nb3Sn magnets exhibited unsatisfactory quench performance and several years of extensive study indicated that the most probable explanation is conductor instability. Recently, the High Field Magnet program attention has been shifted toward instability studies. Strands, cables and magnets were examined in many different ways to search for evidence and characterization of the instabilities.

Flux jump (thermal-electromagnetic phenomena) and mechanical motion are two primary causes of magnet instabilities. If flux jump or mechanical motion occurs in the magnet it will generate a flux change in the coils, which can be detected either by monitoring the coil voltages or the magnetic field. Relatively early in the program we observed that some of the magnets generated unexpectedly high voltage spikes. These spikes were so large that they occasionally tripped our quench detection circuits. On the other hand, the DAQ system was not tuned to study these types of events (large noise level and an amplifier frequency response $<5 \mathrm{kHz}$ ). In order to characterize these voltage spikes a new readout system has been developed at Fermilab. This system is capable of identifying flux changes through the detection of

Manuscript received October 5, 2004. This work was supported in part by the U.S. Department Energy.

Authors are with the Fermi National Accelerator Laboratory, Batavia, Il 60510 USA. voltage spikes up to $30 \mathrm{kHz}$ [1].

Fermilab is also using a "quench antenna" [2] to characterize quench locations in magnet models. Since the quench antenna is sensitive to detect flux changes we used it to look for sudden flux changes likely to be generated by the instabilities.

Although the data from several High Field Magnets has been examined for evidence of voltage spikes only one of them was extensively studied using the new voltage spike detection system since this new system was fully operational only in the summer of 2004. This paper summarizes sudden flux change studies performed with three different high field magnets.

\section{Test SETUP}

For this study data collected in tests of magnets HFDB01, HFDC01 and SR02 were used (details of the magnets and their test results are described elsewhere [3]-[5]). HFDB01, the first 'race track' coil design magnet, HFDC01, the first double aperture race track magnet, and SR02, the second small scale racetrack magnet were all built by Fermilab's High Field Magnet group. Although the mechanical structures of

TABLE I

CONDUCTOR PARAMETERS

\begin{tabular}{lcccc}
\hline \hline \multirow{2}{*}{ Magnets } & Strand & Cable & Jc @ 12 T & RRR \\
\hline HFDB01 & IGC 0.7 mm & 41 strand & $1750 \mathrm{~A} / \mathrm{mm}^{2}$ & 14 \\
HFDC01 & MJR 0.7 mm & 41 strand & $1750 \mathrm{~A} / \mathrm{mm}^{2}$ & 3 \\
SR02 & MJR 1mm & 28 strand & $1750 \mathrm{~A} / \mathrm{mm}^{2}$ & 125 \\
\hline \hline
\end{tabular}

Filament sizes varied between $80-170 \mu \mathrm{m}$

these magnets differ, all utilize $\mathrm{Nb}_{3} \mathrm{Sb}$ conductor. The conductor parameters are summarized in Table I.

Flux changes were collected with three different readout systems.

\section{A. DQD Half Coil Signals}

When the voltage spikes were large enough (over $50 \mathrm{mV}$ ) they triggered our regular quench detection system and data was recorded. The data analyzed are from the "DQD" (digital quench detection) system, which uses a fast, multi-channel ADC to digitize quench detection signals. These data are processed in a VME-based processor where DSP algorithms are used to suppress noise. Since the data have been digitally filtered there is a group delay of 2 milliseconds and frequencies $>300 \mathrm{~Hz}$ are attenuated, changing the 
characteristic shape of the voltage spike signal. The DQD system records four seconds of data prior to the quench trigger. The signals used to look for spikes are the "bucked" half coils where the signal from one half coil is subtracted from the other to cancel common noise. (It should be noted that bucking is inherently less sensitive to voltage signals that occur simultaneously in both half coils. There is obvious cancellation if the signals are same.)

\section{B. Quench Antenna}

The quench antenna is a stationary, three-section probe centered in the warm bore tube of the magnet aperture during test. The middle antenna spans much of the magnet body (coil straight section), while the two end antennas cover the magnet coil lead and return ends, respectively: antenna 1(3) is at the lead (return) end. The antenna coils are based on magnetic measurement probe winding patterns. A dipole coil on one antenna is used for alignment with the main field direction. Small gaps exist between panels for mechanical support. Each probe has four precision windings on coil forms, for detecting normal and skew quadrupole and sextapole field variations caused by current redistribution in the dipole magnet. The time and amplitude of the coil signals are used to determine the radial and azimuthal locations of the disturbance[1]. Signals are conditioned by gain 200 amplifiers and digitized by 16 bit Pentek data loggers operating at $33.3 \mathrm{kHz}$ sample rate when triggered; they capture a data in an interval of a several hundred milliseconds around the triggering event (usually a quench, but spike study triggers have also been used to correlate antenna activity with voltage tap signals).

\section{Voltage spike detection system}

A superconducting magnet voltage transient "spike" detection system has been designed, built, and tested. The

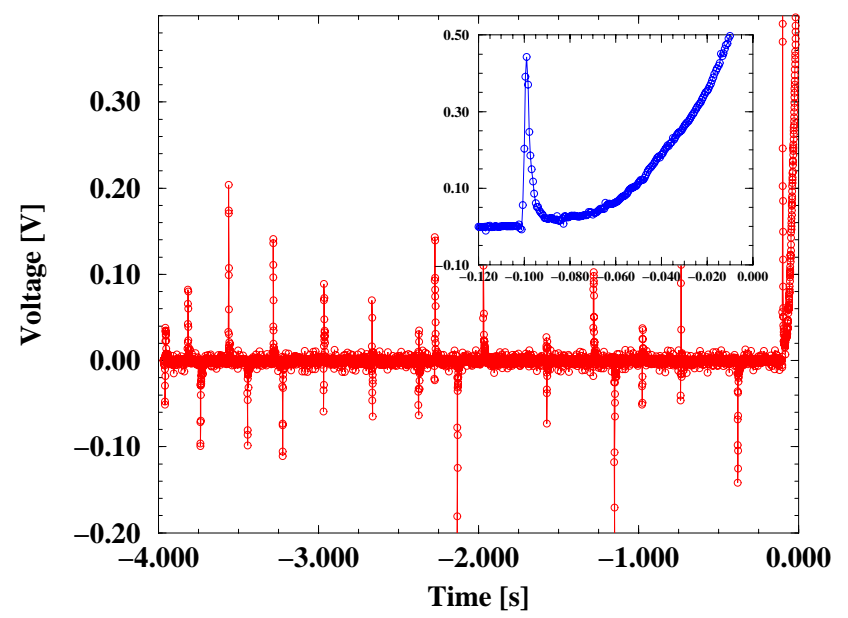

Fig. 1. Typical Voltage Spike is shown occurred in HFDB01 magnet.

system is comprised of three primary hardware components: One, analog filters for anti-aliasing, band limitation, and RF noise rejection; two, amplification and voltage isolation $(1500 \mathrm{~V})$; and three, fast data acquisition and embedded processing. Through modeling, simulation, and testing, the component specifications were optimized for a well-balanced circuit. This was important in order to insure that the phase and magnitude response of the system was matched for optimal bucking of the magnet half coil signals. The DAQ is based on a National Instruments PXI multifunction DAQ: NI PXI-6120 and can sample up to $800 \mathrm{KHz}$ with 16-bit resolution. The software is written in Labview and can perform digital filtering in real-time to reject power supply noise and DC offset in order to improve the signal-to-noise ratio for more confident triggering. A Matlab based stand alone GUI has also been developed for reading and analyzing the resultant data.

\section{TEST RESULTS}

\section{A. Voltage Spikes in HFDB01}

The first attempts at ramps to quench of magnet HFDB01 were interrupted by quench detection system trips at low currents. These trips were found to be due to voltage spikes in the quench detection signals. Upon examination of these data, it was determined that the trips were not accompanied by resistive growth in the magnet and hence these events were not identified as real quenches. The quench detection thresholds were raised to avoid tripping and the test proceeded. When the first real quench was observed, however, it was found to be correlated with the presence of spikes in the voltage signals.

Many of the voltage spikes were recorded ranging from few tens of $\mathrm{mV}$ to few hundreds of $\mathrm{mV}$. These voltage spikes were collected with quench data files. Since no special trigger was developed to capture them, consequently we were able to study only those spikes which occurred within 4 seconds right before the quench. A typical spike is shown in Fig. 1. for a quench when the ramp rate was high: $500 \mathrm{~A} / \mathrm{s}$. This spike plot

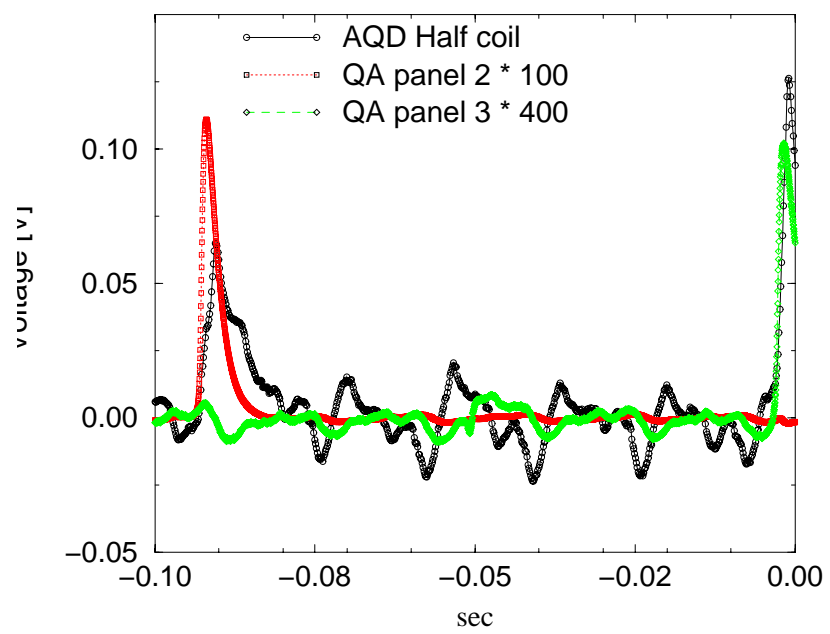

Fig. 2. Two AQD voltage spikes are shown correlated them with quench antenna signals occurred in HFDC01 magnet. Different QA panels picked up the flux change.

displays the DQD half coil balance signal for the 4 second time interval prior to the quench trigger (defined as $\mathrm{t}=0$ in our 
system). In Fig. 1. it can clearly be seen that a voltage spike is associated with the onset of the quench (quench initiation

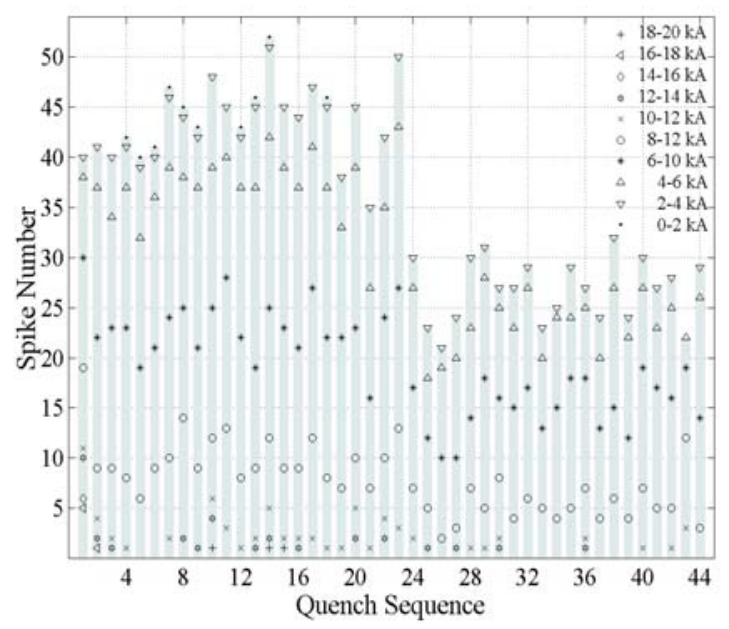

Fig. 3. Voltage spike distribution as function a ramp-to-quench sequence.

region displayed as an inset).

Analyzing the whole data set the following generalizations about "spikes" were concluded:

- Spikes occur on every ramp.

- Spikes occur in both half coils at roughly the same time, but with somewhat different amplitude and detailed time structure.

- Spike frequency and amplitude increase with ramp rate

- Spikes are associated with quench initiation in all plots; in a few cases there is on the order of 20 milliseconds between the spike and quench start; in one case, a spike appears to occur after the quench has begun propagating.

- The magnet current always drops (sometimes dramatically, up to $20 \mathrm{~A}$ in $\leq 1-2 \mathrm{msec}$ ) at the time of a spike; a current increase has not been observed.

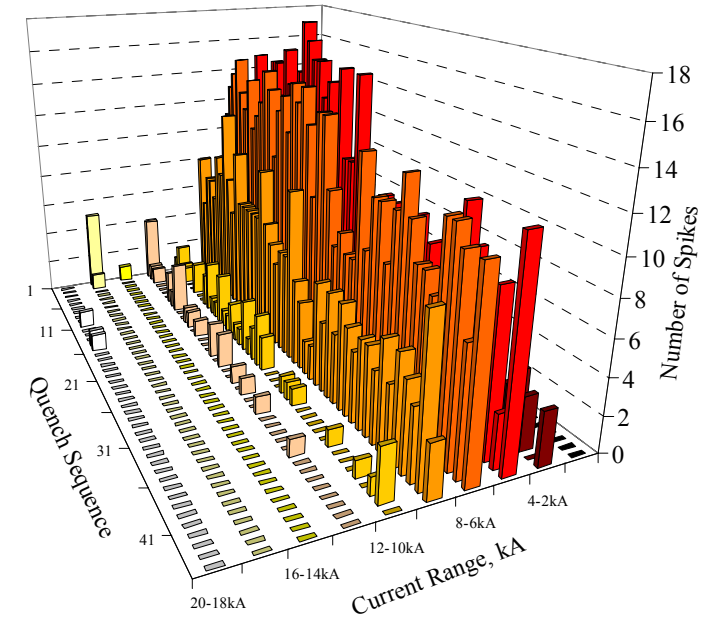

Fig. 4. Voltage spike distribution is plotted binned them in $2 \mathrm{kA}$ bins and as a function of ramp-to-quench sequence.

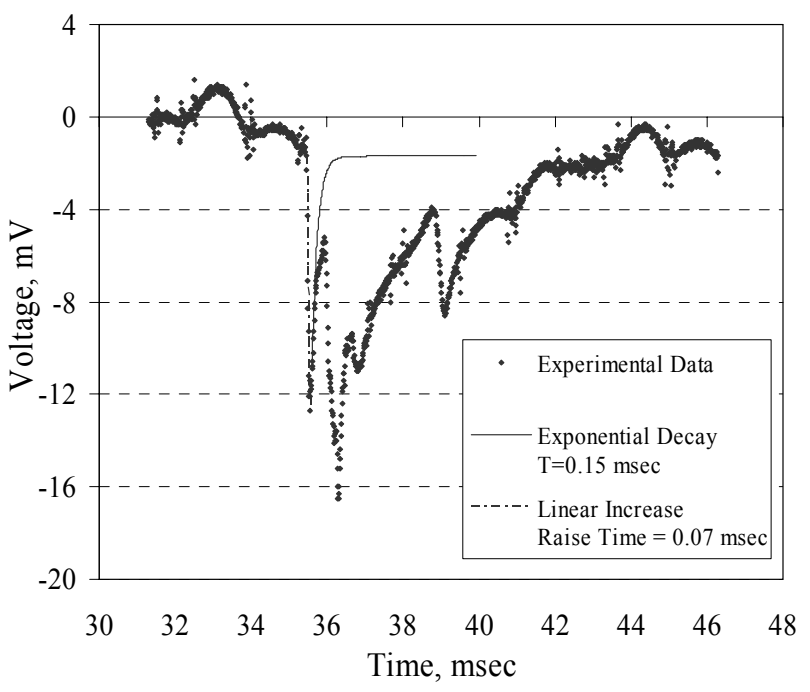

Fig. 5. Typical voltage spike. The first glitch is over-laid with a curve which has a sharp $0.07 \mathrm{msec}$ rise time and an exponential decay with $0.15 \mathrm{msec}$ decay time.

\section{B. Voltage Spikes correlated with QA signals in HFDC01}

During the test of HFDC01 a special "Snapshot" trigger was implemented. "Snapshot" events are triggered by transient excursions in the difference between half coil voltages. The half coil signals were digitized and the data were captured before and after a trigger, in which |Hcoill Hcoil2| exceeded 0.05 or $0.1 \mathrm{~V}$ (the quench detection threshold was $0.25 \mathrm{~V}$ ). Recall that HFDC01 was the first dual aperture race track coil magnets.

One of the magnet apertures contained a quench antenna (QA) so we were able to perform a special test. When snapshot events occurred we read out QA signals as well. On a few occasions we were able to correlate voltage spikes with the QA signal (see Fig. 2.). This was an important test since it proved that these voltage spikes are "real" magnet related

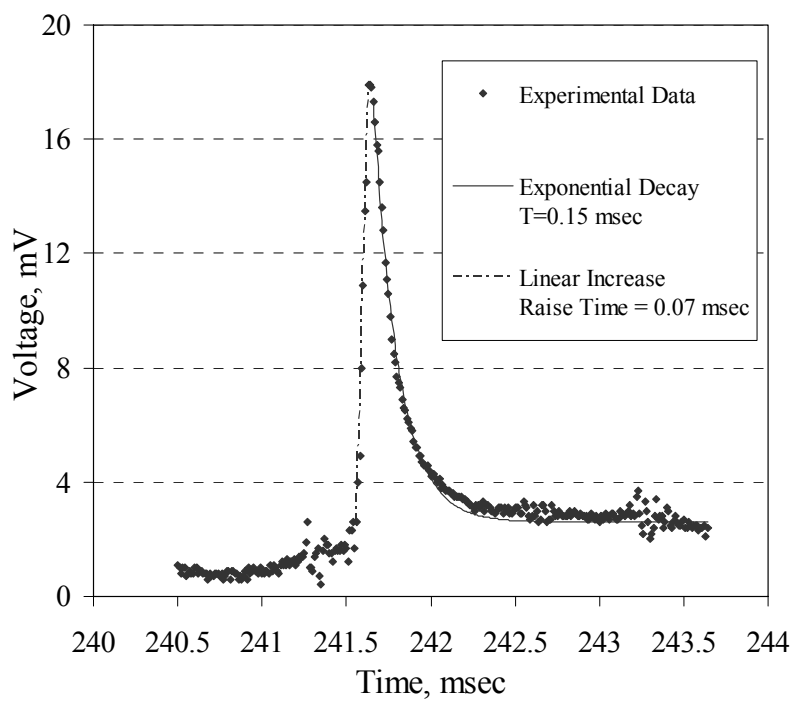

Fig. 6. Most simplest type of voltage spike is plotted. It can be well characterized with a curve which has a $0.07 \mathrm{msec}$ rise time and $0.15 \mathrm{msec}$ exponential decay time. 
events, not just random noise. We also observed that these spikes are not localized, and they were distributed along the cable randomly.

\section{Voltage Spikes in SR02}

SR02 was the first magnet tested when we were able to fully utilize our new Voltage Spike Detection System (VSDS). The run plan of the magnet test requested that each time the magnet current ramped up to initiate a spontaneous quench VSDS was activated to capture voltage spikes. The primary goal was to capture the smallest spikes possible. The power supply regulation was not smooth so the noise magnitude and frequency spectrum was changing with current [6]. In order to capture as many spikes as possible a variable trigger threshold was implemented. The noise was continuously monitored and if the voltage exceeded a 5-sigma value of noise then 0.5 second time interval of data were captured.

Over 1500 voltage spikes were collected during 44 rampto-quench events. In Fig. 3. the number of voltage spikes for different ramp-to-quench events are plotted. Fig 4. also depicts the number of spikes collected as a function of the applied current. After the $22^{\text {nd }}$ quench the number of spikes per ramp didn't change significantly (see Fig.4.). We also noticed that the quench current of SR02 didn't improve much after around the $22^{\text {nd }}-24^{\text {th }}$ quench [3]. This is an indication that some fraction of spikes can be correlated with training of the magnet. We also didn't record any spikes at high current $(>16 \mathrm{kA})$ values after the magnet had been trained.

The voltage spike signals used in this study are the bucked signals of the two half coils. Although bucking the two half coils removed most of the common mode noise the signals were still full of periodic noise so we performed a matched filtering technique to further clean up the signals. A typical voltage spike signal after this clean up is plotted in Fig. 5.,

At low current we observed that some of the events have quite narrow signals and are relatively simple in shape (see Fig. 6.). These simple signals could be well fitted with a curve, which has a sharp rise time $(\sim 0.07 \mathrm{msec})$ followed by an exponential decay with a time constant of $\sim 0.15 \mathrm{msec}$. We also noticed that most of the other spikes have substructure. In Fig. 5. we also over-plotted a "simple" spike normalized to the height of the first 'glitch' of a spike signal. It seems that we could characterize the spike signal quite well as the superposition of many "simple" spikes, with different amplitude and shifted in time. In order to prove this hypothesis we need to re-fine our data analysis.

Some of the voltage spike signals had a completely different shape (see Fig. 7.). These signals appeared only at high current values. The signal looks like rapid oscillations. In Fig. 7 we also plotted the 3-sigma band of the noise. These rapid oscillations are much larger than the span of this band. These signals were correlated with training: during ramping the current to quench, we have seen these events only when the current value became higher than that of the previous quench. These type of signals occurred only when the magnet was exposed to a particular force level for the first time.
In couple of ramp-to-quench, immediately before the quench there was a voltage spike that one can associate with the onset of the quench. This phenomena only happened at the beginning of the magnet training.

\section{CONCLUSIONS}

Three high field magnets built and tested by Fermilab, which utilized multi strand Rutherford type cable made of state-of-the art $\mathrm{Nb}_{3} \mathrm{Sn}$ strands, were examined to look for sudden flux changes. Observed voltage spikes and sudden magnetic field changes both might be linked to magnet

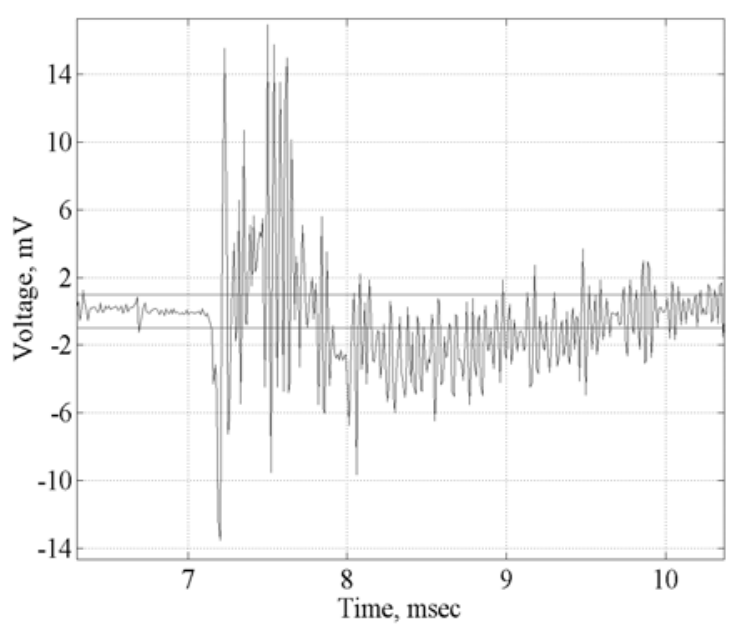

Fig. 7. High frequency spike is plotted observed at high current value.

instabilities. The voltage spike signals were correlated with quench antenna signals, a strong indication that these are magnet phenomena. With the new high-resolution voltage spike detection system we were able to examine the detailed structure of the spikes. Two fundamentally different signal shapes were distinguished, which indicates that they are most likely generated by different mechanisms.

\section{ACKNOWLEDGMENT}

The authors thank the staff of Fermilab's Technical Division who have worked so hard on this effort over the course of the past few years.

\section{REFERENCES}

[1] T. Ogitsu, et al., "Quench Antenna for Superconducting Particle Accelerator Magnets" IEEE Trans. Magnetics, vol 30, 1964, pp. 2273-2276

[2] D. Orris et al., "Voltage Spike Detection in Superconducting .Accelerator Magnets", Submitted to this conference.

[3] S. Feher et al., "Cable Testing for Fermilab's High Field Magnets Using Small Racetrack Coils", Submitted to this conference.

[4] G. Ambrosio, et al.,"Development and test of a Nb3Sn racetrack magnet using the react and wind technology", Advances in Cryogenic Engineering 47A, 2002, pp. 329-336.

[5] V.S. Kashikhin, et al.,"Development and Test of Single-Layer Common Coil Dipole Wound with Reacted Nb3Sn Cable", Proceeding of MT-18 (Morioca, Japan).

[6] Carcagno et al., "Ripple Current Effects on the Performance of Superconducting High Field Magnets using a New 30 kA Power System at Fermilab", Submitted to this conference. 
